Nobuo Yaegashi • Masato Senoo - Shigeki Uehara

Hisako Suzuki • Tohru Maeda · Keiya Fujimori

Fumiki Hirahara • Akira Yajima

\title{
Age-specific incidences of chromosome abnormalities at the second trimester amniocentesis for Japanese mothers aged 35 and older: collaborative study of 5484 cases
}

Received: July 23, 1997 / Accepted: December 1, 1997

\begin{abstract}
The aim of this study was to calculate the expected incidences of chromosome abnormalities found at amniocentesis in Japanese women aged 35 and older. From four clinics in Japan, we gathered genetic amniocentesis data on 5484 pregnant women at risk only due to their advanced age, 35 years and older. We analyzed the data using the logistic regression model. Of the 5484 fetuses, 117 $(2.1 \%)$ were diagnosed with a chromosome abnormality. The abnormal karyotypes included 42 cases of trisomy $21 ; 13$ of trisomy $18 ; 7$ of trisomy $13 ; 10$ of $47, \mathrm{XXY} ; 4$ of $47, X X X ; 1$ of 47,XYY; 27 with various structural aberrations; and 13 with various types of mosaicism. The incidences of trisomy 21, lethal autosomal aneuploidies (trisomy 18 and trisomy 13), and sex-chromosome abnormalities (XXY, XXX, XYY) increased with maternal age. Parameters of the regression equations with their standard errors were calculated and the expected incidences of chromosome abnormalities at each maternal age were derived. The expected incidences of chromosome abnormalities obtained in this study are the first data published for Japan and will be useful for the counseling of pregnant women. The incidence of trisomy 21 is not different from the rates published previously for Western countries. The incidences of chromosome abnormalities are not affected by race or by geographic factors.
\end{abstract}

N. Yaegashi $\cdot$ M. Senoo $\cdot$ S. Uehara $(\bowtie) \cdot$ H. Suzuki $\cdot$ A. Yajima Department of Obstetrics and Gynecology, Tohoku University School of Medicine, 1-1 Seiryo-machi, Aoba-ku, Sendai 980-8574, Japan

Tel. +81-22-717-7254; Fax +81-22-717-7258

e-mail: nyaegashi@gonryo.med.tohoku.ac.jp

T. Maeda

Department of Obstetrics and Gynecology, Kitazato University

School of Medicine, Sagamihara, Japan

K. Fujimori

Department of Obstetrics and Gynecology, Fukushima Prefectural

College of Medicine, Fukushima, Japan

F. Hirahara

Department of Obstetrics and Gynecology, Yokohama City

University of Medicine, Yokohama, Japan
Key words Advanced maternal age - Amniocentesis · Prenatal diagnosis $\cdot$ Chromosome $\cdot$ Down syndrome

\section{Introduction}

The frequencies of Down syndrome (trisomy 21) and other chromosome abnormalities are well known to increase with advancing maternal age (Polani et al. 1976; Schreinemachers et al. 1982; Ferguson-Smith and Yates 1984; Hook et al. 1984). Obstetricians, family practitioners, and clinical geneticists are queried by pregnant women concerning the possibility that their offspring may have a cytogenetic abnormality. Indeed, even if not queried, the failure by a physician to provide such information in putatively high-risk circumstances to pregnant women has been cited as grounds for legal liability. During genetic counseling before prenatal testing, it is important to provide pregnant women with an accurate estimate of the risks of abnormalities. Especially for older women, doctors predict the incidence of each chromosomal aberration at the patient's age and get the patient's informed consent before testing. To detect chromosome abnormalities, genetic amniocentesis is commonly carried out at midtrimester as one of several testing procedures for prenatal diagnosis, since it is highly accurate and safe, and does not increase the risk of fetal loss or injury (The NICHD National Registry for Amniocentesis Study Group, 1976).

In Japan, the demand for genetic counseling and prenatal tests has increased markedly, because the number and proportion of pregnancies in women aged 35 years and older has increased over a 20 -year period to reach $10 \%$ of all live births in 1995 (Mother's and Children's Health Organization 1996). However, the data employed during counseling on estimated risk of chromosome abnormalities are mainly obtained from Western countries, such as Europe, North America, and Australia. Since biological and environmental differences may influence the incidences of chromosome aberrations, it appeared worthwhile to compile available data, derived from Japanese mothers, to 
estimate the rates of all significant cytogenetic abnormalities by 1-year maternal age interval.

In this report, we obtained from four genetic clinics in Japan a pool of genetic amniocentesis data from tests performed in midtrimester only due to advanced maternal age. We analyzed the relationship of maternal age to the incidences of cytogenetic abnormalities by using logistic regression, and estimated the incidence of abnormalities by maternal age at amniocentesis among Japanese.

\section{Materials and methods}

We collected data on Japanese cases of cytogenetic amniocentesis from four genetic clinics, Kitazato University Hospital, Fukushima Prefectural University Hospital, Yokohama City University Hospital, and Tohoku University Hospital, where obstetricians routinely carried out cytogenetic amniocentesis during the second trimester of pregnancy (from the 14th to the 18th week), and pooled the results. The gestational age was estimated from the last menstrual period and corrected by ultrasound measurement of the fetus. Contributors were asked to provide information on pregnancies tested solely on account of maternal age and to exclude pregnancies which had additional chromosomal indications such as a previous chromosome abnormality, a parental chromosomal translocation, and so on. Only those cases where the maternal age was 35 or older at the time of amniocentesis were included in this study. Data included the number of prenatal cytogenetic amniocenteses performed, and the type and the number of chromosomal abnormalities according to each maternal age.

Amniocytes were cultured in Ham F10 or Chang's media containing $10 \%$ or $15 \%$ fetal bovine serum under $5 \% \mathrm{CO}_{2}$ at $37^{\circ} \mathrm{C}$ for more than 2 weeks. After colcemid was added, the cells were harvested after an additional 5-h culture, treated in hypotonic $\mathrm{KCl}$ solution $(75 \mathrm{mM})$, fixed in methanol/acetic acid fixative (3:1), and deposited on glass slides.
After the glass slides were air-dried or mechanically dried, G-banding was carried out using a standard method.

Chromosome abnormalities studied herein include aneuploidies, structural aberrations such as translocations and inversions, and mosaicisms not only on autosomes but also on sex-chromosomes. However, normal variants of chromosome structures, such as pericentric inversion of chromosome 9, enlarged heterochromatin on various chromosomes, enlarged satellites, and so on, were eliminated. When mosaicisms were unclear as to whether they were artifacts or not, a second amniocentesis or fetal blood sampling was carried out for certainty.

To analyze the relationship between the maternal age at amniocentesis and the logarithm of the incidences of chromosome abnormalities, logistic regression analysis was undertaken using the formula $y=\exp (a+b x)$, where $x$ is the maternal age at amniocentesis and $y$ is the incidence of the cytogenetic abnormalities at age $x$. The values for the parameters $a$ and $b$ were obtained by a maximum likelihood regression approach. The correlation was assessed to be statistically significant when the maternal age coefficient $b$ was significantly different from zero $(P<0.05)$ when the standard error was taken into consideration. The equation $y=\exp (a+b x)$ was used to calculate the expected incidence of chromosome abnormalities at amniocentesis by 1year maternal age intervals.

\section{Results}

Table 1 shows chromosomal abnormalities in surveys of 5484 amniocenteses in the second trimester in pregnancies monitored due to maternal age $>35$ years. Of these, 117 cases were diagnosed to have a chromosome abnormality, which resulted in an overall incidence of chromosome abnormalities of $2.1 \%(117 / 5484)$. Nonmosaic autosome and sex-chromosome aneuploidies totaled 62 (42 cases of trisomy 21,13 of trisomy 18 , and 7 of trisomy 13 ) and 15 (10 of

Table 1 Number and incidence of chromosome abnormality by maternal age

\begin{tabular}{|c|c|c|c|c|c|c|c|c|c|c|}
\hline \multirow{2}{*}{$\begin{array}{l}\text { Maternal age at } \\
\text { amniocentesis }\end{array}$} & \multirow{2}{*}{$\begin{array}{l}\text { No. of } \\
\text { fetuses }\end{array}$} & \multicolumn{7}{|c|}{ Aneuploidies, excluding mosaicism } & \multirow[t]{2}{*}{ Others } & \multirow[t]{2}{*}{ Total } \\
\hline & & $47,+21$ & $47,+18$ & $47,+13$ & XXY & $\mathrm{XXX}$ & XYY & $\begin{array}{l}\text { All } \\
\text { aneuploidies }\end{array}$ & & \\
\hline 35 & 736 & $1(1.36)^{\mathrm{a}}$ & & & & & & $1(1.36)$ & $1(1.36)$ & $2(2.72)$ \\
\hline 36 & 850 & $4(4.71)$ & $2(2.35)$ & & $2(2.35)$ & & & $8(9,41)$ & $4(4.71)$ & 12 (14.12) \\
\hline 37 & 824 & $8(9.71)$ & & & & & & $8(9.71)$ & $4(4.85)$ & $12(14.56)$ \\
\hline 38 & 800 & $2(2.50)$ & & $2(2.50)$ & & $2(2.50)$ & & $6(7.50)$ & $7(8.75)$ & $13(16.25)$ \\
\hline 39 & 713 & $8(11.22)$ & & $2(2.81)$ & $1(1.40)$ & & $1(1.40)$ & $12(19.17)$ & $8(11.22)$ & $20(28.05)$ \\
\hline 40 & 626 & $6(9.58)$ & $5(7.99)$ & $1(1.60)$ & $1(1.60)$ & & & $13(20.77)$ & $10(15.97)$ & $23(36.74)$ \\
\hline 41 & 417 & $3(7.19)$ & $3(7.19)$ & $2(4.80)$ & $2(4.80)$ & & & $10(23.98)$ & $1(2.40)$ & $11(26.38)$ \\
\hline 42 & 255 & $6(23.53)$ & $1(3.92)$ & & $3(11.76)$ & $1(3.92)$ & & $11(43.14)$ & $2(7.84)$ & $13(50.98)$ \\
\hline 43 & 146 & $2(13.70)$ & $1(6.85)$ & & $1(6.85)$ & $1(6.85)$ & & $5(34.23)$ & $2(13.70)$ & $7(47.95)$ \\
\hline 44 & 87 & $2(22.99)$ & & & & & & $2(22.99)$ & $1(11.49)$ & $3(34.48)$ \\
\hline 45 & 22 & & $1(45.45)$ & & & & & $1(45.45)$ & & $1(45.45)$ \\
\hline 46 & 7 & & & & & & & & & \\
\hline 47 & 1 & & & & & & & & & \\
\hline Total & 5484 & $42(7.66)$ & $13(2.37)$ & $7(1.28)$ & $10(2.82)$ & $4(0.73)$ & $1(0.18)$ & 77 (14.04) & $40(7.29)$ & 117 (21.33) \\
\hline
\end{tabular}

${ }^{a}$ Number (and incidences per 1000). 
Table 2 Coefficients and standard errors for logistic regression calculations*

\begin{tabular}{|c|c|c|c|c|c|c|}
\hline & \multicolumn{2}{|c|}{ Constant term } & \multicolumn{4}{|c|}{ Maternal term } \\
\hline & $a$ & $\mathrm{SE}$ & $b$ & SE & $95 \% \mathrm{CI}$ & $P$ value \\
\hline Trisomy 21 & -7.605 & 2.485 & 0.245 & 0.063 & $0.100-0.389$ & 0.0045 \\
\hline$+18,+13$ & -9.661 & 3.464 & 0.284 & 0.085 & $0.075-0.493$ & 0.0159 \\
\hline Sex chromosomes & -10.087 & 4.196 & 0.290 & 0.105 & $0.020-0.560$ & 0.0400 \\
\hline All aneuploidies** & -7.719 & 2.085 & 0.261 & 0.052 & $0.144-0.379$ & 0.0007 \\
\hline
\end{tabular}

95\% CI: 95 percent confidence interval.

* Model y $=\exp (a+b x)$ where $x=$ maternal age in years at time of amniocentesis and $y=$ incidence of the chromosome abnormality.

**Excluding mosaicisms and structural abnormalities.

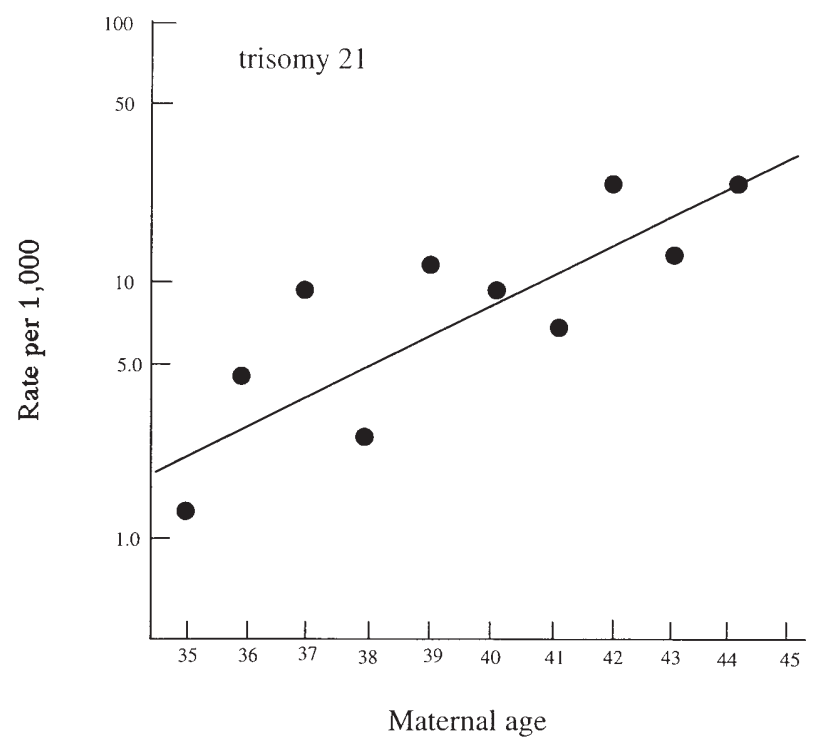

Fig. 1 Proportion (per 1000 fetuses) of trisomy 21 (ordinate) and maternal age (abscissa)

$\mathrm{XXY}, 4$ of XXX, and 1 of XYY), respectively. No cases of $\mathrm{X}$-monosomy (Turner syndrome) were diagnosed.

For trisomy 21 , the maternal age coefficient $b$ was positive $(b=0.245)$, and significantly different from zero $(95 \%$ confidence interval (CI): $0.100-0.389, P=0.0045)$ when the standard error $(\mathrm{SE}=0.063)$ was taken into consideration, showing that the incidence of trisomy 21 increases exponentially with advancing maternal age (Fig. 1, Table 2).

For trisomy 18 and trisomy 13, increasing incidences in older mothers for each trisomy were observed, similar to that for trisomy 21 , but the relationships were not statistically significant. When these two aneuploidies were calculated together as a lethal autosomal aneuploidy, the incidence increased with advancing maternal age $(b=0.284, \mathrm{SE}=0.085,95 \%$ CI: $0.075-0.493, P=0.0159)$ (Fig. 2, Table 2).

For each sex-chromosome aneuploidy, a tendency to increasing incidences in older mothers was noted, similar to that of trisomy 21, but the correlations were not statistically significant. When three types of sex-chromosome aneuploidy were calculated together as sex-chromosome aneuploidies, the incidences significantly increased with

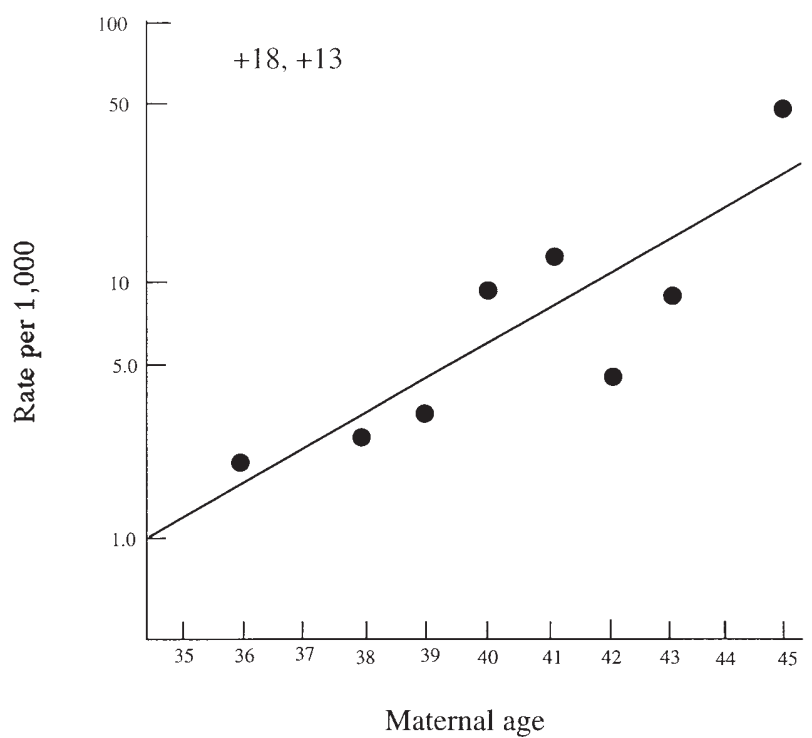

Fig. 2 Proportion (per 1000 fetuses) of lethal autosomal aneuploidies (trisomy 18 and trisomy 13) (ordinate) and maternal age (abscissa)

advancing maternal age $(b=0.290, \quad \mathrm{SE}=0.105, \quad 95 \%$ CI: $0.020-0.560, P=0.040$ ) (Fig. 3, Table 2).

For all aneuploidies, the maternal age coefficient $b$ was also positive $(b=0.261)$, and significantly different from zero (95\% CI: $0.144-0.379, P=0.0007)$ when the standard error $(\mathrm{SE}=0.052)$ was taken into consideration (Fig. 4, Table 2). No nonmosaic trisomies other than the six types listed were detected.

Based on these regression analyses, we calculated the expected incidences of trisomy 21, lethal autosomal aneuploidies (trisomy 18 and trisomy 13), sex-chromosome aneuploidies (47,XXY; 47,XXX; 47,XYY), and all aneuploidies, excluding mosaicism and structural aberrations, at the midtrimester amniocentesis among Japanese (Table 3). There were no significant differences between the coefficients obtained in this study and those from the previous studies from other countries (Polani et al. 1976; Schreinemachers et al. 1982; Ferguson-Smith and Yates 1984; Hook et al. 1984) by analysis of covariance; for example, the equation for trisomy $21, y=\exp (-14.7+$ $0.262 x$ ), obtained from North American studies (Hook et al. 1984). 


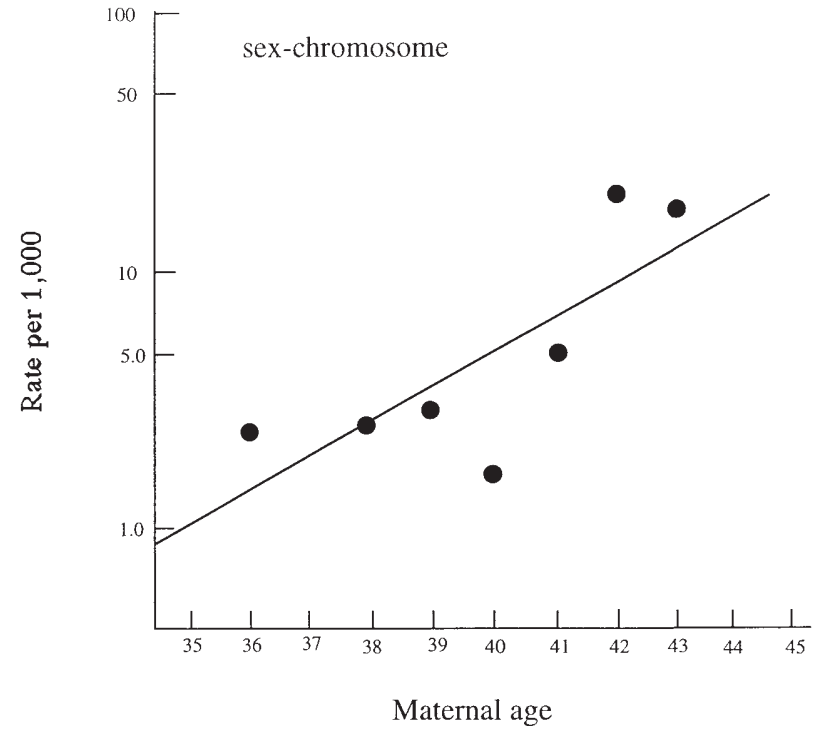

Fig. 3 Proportion (per 1000 fetuses) of sex-chromosome abnormalities (XXY, XXX, XYY) (ordinate) and maternal age (abscissa)

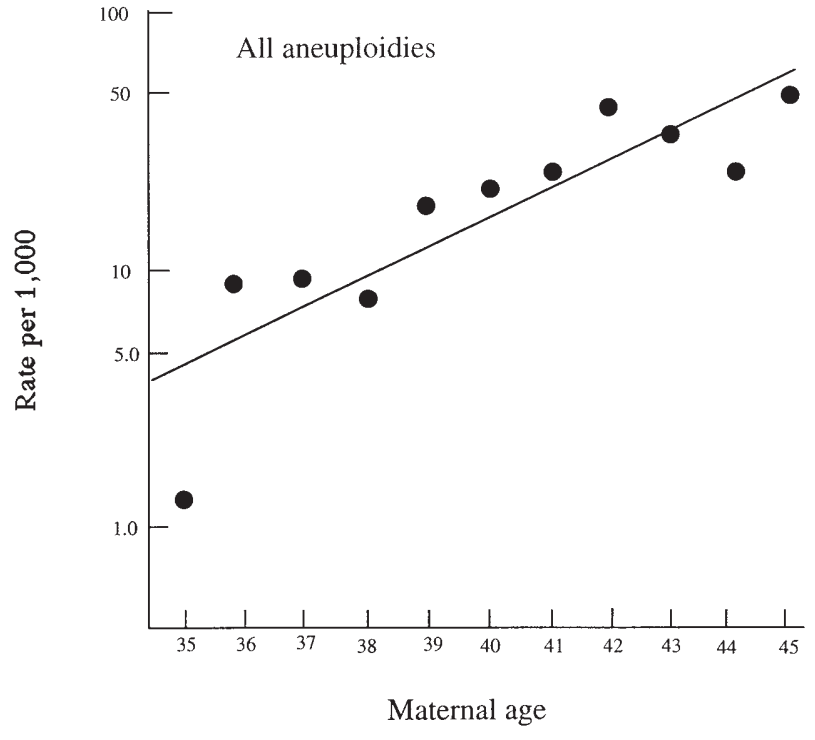

Fig. 4 Proportion (per 1000 fetuses) of all aneuploidies (ordinate), excluding mosaicisms and structural aberrations, and maternal age (abscissa)

Table 3 Regression-smoothed expected incidence of abnormality /1000 at amniocentesis by maternal age

\begin{tabular}{llccc}
\hline $\begin{array}{l}\text { Maternal age at } \\
\text { amniocentesis }\end{array}$ & \multicolumn{4}{l}{ Regression-smoothed expected incidence (per 1000) } \\
\cline { 2 - 5 } & Trisomy 21 & $+18,+13$ & Sex chromosomes & All aneuploidies \\
\hline 35 & 2.62 & 1.32 & 1.07 & 4.12 \\
36 & 3.37 & 1.76 & 1.42 & 5.35 \\
37 & 4.31 & 2.33 & 1.90 & 6.94 \\
38 & 5.50 & 3.10 & 2.54 & 9.02 \\
39 & 7.03 & 4.12 & 3.40 & 11.70 \\
40 & 8.98 & 5.47 & 4.54 & 19.20 \\
41 & 11.47 & 7.26 & 6.07 & 25.61 \\
42 & 14.66 & 9.65 & 8.11 & 33.25 \\
43 & 18.73 & 12.82 & 10.84 & 43.16 \\
44 & 23.93 & 17.03 & 14.48 & 56.04 \\
45 & 30.57 & 22.62 & 19.36 & \\
\hline
\end{tabular}

All other clinically significant cytogenetic diagnoses are shown in Table 4 . The total number of structural aberrations was 27 ( 1 of unbalanced Robertsonian translocation, 10 of balanced reciprocal translocation, 4 of balanced Robertsonian translocation, and 12 of inversion). The numbers of mosaic autosome aneuploidies and those of sex-chromosome aneuploidies were 11 and 2, respectively. In the cases with a mosaic autosome trisomy, aneuploidies of chromosomes other than chromosomes 21,18 , and 13 were diagnosed.

For other chromosome abnormalities including translocations, inversions, and mosaicisms, no significant relationship was found between the maternal age and incidence.

\section{Discussion}

The present study revealed the incidences of chromosome abnormalities at amniocentesis by maternal age in Japan.
These are the first published data from a country or region other than Western countries. Regression analysis clearly showed that the incidences of trisomy 21, lethal autosome abnormalities, and sex-chromosome aneuploidies increased with advancing maternal age, as was shown in the previous reports (Polani et al. 1976; Schreinemachers et al. 1982; Ferguson-Smith and Yates 1984; Hook et al. 1984; Hassold et al. 1996). In addition, we obtained an equation for the expected incidence of each chromosome abnormality. These data will be useful for the genetic counseling of Japanese women.

We calculated the parameters of the equation $[y=\exp (a+b x)]$ (Table 2), and the smoothed expected rates derived from the equations of trisomy 21 , lethal autosome trisomies (trisomy 18 and trisomy 13), sexchromosome aneuploidies, and all aneuploidies at each maternal age 35 years and older (Table 3 ). Comparing the equation for trisomy $21[y=\exp (-7.6+0.245 x]$ obtained from the present study with that $[y=\exp (-14.7+0.262 x)]$ 
Table 4 Other chromosome abnormalities by maternal age

\begin{tabular}{|c|c|c|c|c|c|c|c|}
\hline \multirow{3}{*}{$\begin{array}{l}\text { Maternal age at } \\
\text { amniocentesis }\end{array}$} & \multicolumn{3}{|l|}{ Translocation } & \multirow[t]{3}{*}{ Inversion } & \multicolumn{2}{|l|}{ Mosaicism } & \multirow[t]{3}{*}{ Total } \\
\hline & \multirow{2}{*}{$\frac{\text { Unbalanced }}{\text { Robertsonian }}$} & \multicolumn{2}{|l|}{ Balanced } & & \multirow[t]{2}{*}{ Autosomal } & \multirow[t]{2}{*}{$45, \mathrm{X} / 46, \mathrm{XY}$} & \\
\hline & & Reciprocal & Robertsonian & & & & \\
\hline 35 & & $1(1.36)^{\mathrm{a}}$ & & & & & $1(1.36)$ \\
\hline 36 & & $1(1.18)$ & $1(1.18)$ & $1(1.18)$ & $1(1.18)$ & & $4(4.71)$ \\
\hline 37 & & $3(3.64)$ & & $1(1.21)$ & & & $4(4.85)$ \\
\hline 38 & $1(1.25)$ & $2(2.50)$ & & $2(2.50)$ & $1(1.25)$ & $1(1.25)$ & $7(8.75)$ \\
\hline 39 & & $1(1.40)$ & & $5(7.01)$ & $2(2.81)$ & & $8(11.22)$ \\
\hline 40 & & $2(3.19)$ & $2(3.19)$ & $1(1.60)$ & $4(6.39)$ & $1(1.60)$ & 10 (15.97) \\
\hline 41 & & & & $1(2.40)$ & & & $1(2.40)$ \\
\hline 42 & & & $1(3.92)$ & & $1(3.92)$ & & $2(7.84)$ \\
\hline 43 & & & & $1(6.85)$ & $1(6.85)$ & & $2(13.70)$ \\
\hline 44 & & & & & 1 (11.49) & & 1 (11.49) \\
\hline Total & $1(0.18)$ & $10(1.82)$ & $4(0.73)$ & $12(2.19)$ & $11(2.01)$ & $2(0.36)$ & $40(7.29)$ \\
\hline
\end{tabular}

${ }^{\mathrm{a}}$ Number (and incidences per 1000).

obtained from European and North American studies (Hook et al. 1984), there were no significant differences in the parameters, which suggests that the prevalence of trisomy 21 is not affected either by race or by geographic factors. Recent studies indicated that aneuploidy originating not only in meiosis-I but also meiosis-II correlates to maternal age (Plachot et al. 1988; Macas et al. 1990; Dailey et al. 1996; Hassold et al. 1996). Based on these facts, we suggest that nondisjunction may be caused by factors induced only by aging and may be a universal phenomenon in human reproduction.

Since cytogenetic abnormalities prenatally diagnosed by amniocentesis show a higher rate of spontaneous abortion and/or stillbirth than cytogenetically normal fetuses (Hook 1983; Hook et al. 1988, 1989; Halliday et al. 1995; Macintosh et al. 1995), the incidence at prenatal testing is markedly different from that at live birth. Compared to the previous reports of nonmosaic aneuploidies diagnosed in live births (Hook et al. 1984; Mutton et al. 1991), the abnormal karyotypes and their order revealed in this study were almost the same, with some exceptions, but the incidences of each nonmosaic aneuploidy were markedly higher; for example, the incidence of trisomy 21 in Japan is 1.42 / 1000 live births (Maeda et al. 1991). These higher incidences may be caused by selective miscarriage occurring during the second or third trimester of pregnancy, and the fetus with the aneuploidy may be stillborn. During counseling, the estimated risks obtained from the prenatal testing data should be discussed, and the counselor should also provide an estimate of the contribution of chromosome abnormalities to spontaneous fetal death.

In all of the nonmosaic aneuploidies diagnosed at the second trimester amniocentesis, only six kinds of trisomies were detected. Although X monosomy and trisomy 16 were detected as aneuploidies in chorionic villus sampling (CVS) (Hook et al. 1988) or in early amniocentesis (Lockwood and Neu 1993), there were no such aneuploidies detected in this study. Since cytogenetic CVS and early amniocentesis are usually carried out in the first trimester of pregnancy (CVS in the 9th to the 11th gestational week; early amniocentesis in the 11th to the 14th gestational week), most fetuses with those aneuploidies may have been spontaneously miscarried before the second trimester amniocentesis. If additional cases are studied, some such aneuploidies may be detected at the second trimester amniocentesis as exceptional cases.

The proportion of pregnant women age 35 or over has been gradually increasing, and now constitutes more than $10 \%$ of all pregnant women in Japan (Mother's and Children's Health Organization 1996). These women will receive information about the high risk of chromosome abnormalities due to advanced age from the mass media or medical sources, and will visit obstetrical clinics to receive genetic counseling or ask for prenatal testing. If pregnant women of advanced age have no information about the risks, obstetricians must explain them. During genetic counseling for women at risk due to their advanced age, obstetricians or other healthcare professionals will need to present the estimated possibility of chromosome abnormalities, and let patients choose whether they want to have prenatal testing on the basis of this possibility. We believe that the possibility should be derived from the data at the time of testing, such as the expected rates revealed in this study.

The present study provides informative data for the counseling of Japanese women. However, more cases should be examined further to obtain greater accuracy in predicting the rates of each chromosome abnormality at midtrimester amniocentesis.

\section{References}

Dailey T, Dale B, Cohen J, Munne S (1996) Association between nondisjunction and maternal age in meiosis-II human oocytes. Am J Hum Genet 59: 176-184

Ferguson-Smith MA, Yates JRW (1984) Maternal age specific rates for chromosome aberrations and factors influencing them: Report of a collaborative European study on 52,965 amniocenteses. Prenat Diagn 4: 5-44

Halliday JL, Watson LF, Lumley J, Danks DM, Sheffield LJ (1995) New estimates of Down syndrome risks at chorionic villus sampling, 
amniocentesis, and livebirth in women of advanced maternal age from a uniquely defined population. Prenat Diagn 15: 455-465

Hassold T, Abruzzo M, Adkins K, Griffin D, Merrill M, Millie E, Sakar D, Shen J, Zaragoza M (1996) Human aneuploidy incidence, origin, and etiology. Environ Mol Mutagen 28: 167-175

Hook EB (1983) Chromosome abnormalities and spontaneous fetal death following amniocentesis: Further data and associations with maternal age. Am J Hum Genet 35: 110-116

Hook EB, Cross PK, Regal RR (1984) The frequency of 47,+21, $47,+18$, and $47,+13$ at the uppermost extremes of maternal ages: results on 56,094 fetuses studied prenatally and comparisons with data on livebirths. Hum Genet 68: 211-220

Hook EB, Cross PK, Jackson L, Pergament E, Brambati B (1988) Maternal age-specific rates of $47,+21$ and other cytogenetic abnormalities diagnosed in the first trimester of pregnancy in chorionic villus biopsy specimens: Comparison with rates expected from observations at amniocentesis. Am J Hum Genet 42: 797-807

Hook EB, Topol BB, Cross PK (1989) The natural history of cytogenetically abnormal fetuses detected at midtrimester amniocentesis which are not terminated electively: New data and estimates of the excess and relative risk of late fetal death associated with $47,+21$ and some other abnormal karyotypes. Am J Hum Genet 45: 855-861

Lockwood DH, Neu RL (1993) Cytogenetic analysis of 1375 amniotic fluid specimens from pregnancies with gestational age less than 14 weeks. Prenat Diagn 13: 801-805

Macas E, Floersheim Y, Hotz E, Imthurn B, Keller PJ, Walt H (1990) Abnormal chromosomal arrangements in human oocytes. Hum Reprod 5: 703-707
Macintosh MCM, Wald NJ, Chard T, Hansen J, Mikkelsen M, Therkelsen AJ, Petersen GB, Lundsteen C (1995) Selective miscarriage of Down's syndrome fetuses in women aged 35 years and older. Br J Obstet Gynaecol 102: 798-801

Maeda T, Ohno M, Matsunobu A, Yoshihara K, Yabe N (1991) A cytogenetic survey of 14,835 consecutive liveborns. Jpn J Human Genet 36: 117-129

Mother's and Children's Health Organization (1996) Live births by age of mother. In: Mother's and Children's Health and Welfare Association (ed) Maternal and child health statistics of Japan 1996. Tokyo, pp 47-53

Mutton DE, Alberman E, Ide R, Bobrow M (1991) Results of first year (1989) of a national register of Down's syndrome in England and Wales. Br Med J 303: 1295-1297

The NICHD National Registry for Amniocentesis Study Group (1976) Midtrimester amniocentesis for prenatal diagnosis: Safety and accuracy. JAMA 236: 1471-1476

Plachot M, de Grouchy J, Junca AM, Mandelbaum J, Salat-Baroux J, Cohen J (1988) Chromosome analysis of human oocytes and embryos: does delayed fertilization increase chromosome imbalance? Hum Reprod 3: 125-127

Polani PE, Alberman E, Berry AC, Blunt S, Singer JD (1976) Chromosome abnormalities and maternal age. Lancet II: 516-517

Schreinemachers DM, Cross PK, Hook EB (1982) Rates of trisomies $21,18,13$ and other chromosome abnormalities in about 20,000 prenatal studies compared with estimated rates in live births. Hum Genet 61: 318-324 